\title{
The role of the future physician: building on shifting sands
}

\author{
Authors: Linford Fernandes, ${ }^{A}$ Michael EB FitzPatrick ${ }^{B}$ and Matthew Roycroft ${ }^{C}$
}

The role of the future physician in the NHS is of interest to current doctors, patients, policymakers and the wider public. Amid the COVID-19 pandemic, it has never been more clear that the healthcare needs of the population, and the technical and scientific advances with which to solve them, are rapidly evolving and the medical workforce must adapt to these changes to deliver personalised healthcare. This article considers the current challenges that need to be addressed to deliver a future physician-led healthcare service that works for its patients. Key themes are expanded upon, including the changing healthcare workforce, digital and technological innovation, service delivery, complex conditions and changing patient demographics. The impact and challenges of the ongoing COVID-19 pandemic on these factors are highlighted. Avenues for development are suggested, both in postgraduate medical training and the health service as a whole. These changes will be required to deliver the physicians of the future, imbued with the skills and attributes they will need to provide a high standard of care in the mid-21st century.

KEYWORDS: future physician, NHS long term plan, flexible working, workforce, digital health, COVID-19

DOI: $10.7861 /$ clinmed.2020-0030

\section{Introduction}

Medicine is changing at an unprecedented pace, driven by social, technological and scientific changes. How will this healthcare revolution change the role of the physician in the UK in the coming decades? Health Education England last year called for evidence on the expectations of patients and the public of 21st century doctors, which will bring to light a range of views on how medicine will need to adapt to meet the population needs. ${ }^{1}$ At the time of the concept of this article, the severe acute respiratory syndrome coronavirus 2 (SARS-CoV-2) epidemic, known as COVID-19, had not emerged and yet in just a few short months, healthcare systems in the UK and the world have had to significantly alter their delivery

Authors: Aneurology clinical research fellow, Leeds Teaching Hospitals NHS Trust; ${ }^{B}$ gastroenterology clinical research fellow, University of Oxford; 'ST7 geriatric medicine, Sheffield Teaching Hospitals NHS Foundation Trust. All authors contributed equally to the manuscript. of healthcare to limit the human cost of this pandemic. What legacy will the SARS-CoV-2 pandemic leave on the physician role? In this article, we identify common themes that physician trainees perceive as the key drivers for change in the future medical workforce, and consider how best to imbue the physicians of the future with the knowledge, skills, and expertise they will need to thrive in medicine in the mid-21st century (see Fig 1).

The future workforce: blended, multiprofessional and flexible

The role of the future doctor cannot be considered in isolation, as it will be shaped by changes to the wider healthcare workforce. Doctors will continue to be expected to lead the care of patients, as they do now, and work in collaboration with allied healthcare professionals to deliver holistic care. The roles of medical associate professionals (MAPs), such as advanced care practitioners (ACPs), advanced nurse practitioners (ANPs) and physician associates (PAs), will evolve in parallel, and that evolution should complement the physician's role, allowing doctors to work 'at the top of their licence'. Employers will expect flexibility in the roles of doctors over their careers, with ongoing training and skills development required as medicine changes more rapidly. The introduction of regulated frameworks for such skills development, such as proposals for credentialing by the GMC, will allow this flexibility over a physician's career, and fill skills gaps rapidly. These credentials are qualifications aimed to provide recognition for a standardised set of skills achieved in currently discrete areas of clinical work which have a growing patient need: for example, thrombectomy in hyperacute ischaemic stroke. ${ }^{2}$

An engaged workforce will develop a sense of agency and help deliver better value healthcare. Employers will need to work closely with doctors to provide them with the resources and support they require to deliver value-based healthcare responsive to local population needs. ${ }^{3}$ This will include adapting to the changes in desired working practices by physicians in training and beyond, with a shift to fully flexible working practices allowing clinicians to have more control over working patterns. As physicians focus more on clinical complexity and the cognitive load that such practice imposes, employers must ensure they have the time and space needed to ensure fatigue and burnout do not develop.

The current healthcare workforce shortages are well documented. Future healthcare provision will be resource-intensive and require high staff numbers, but what proportion of these will be doctors is less clear. While MAPs, such as PAs, work well in single-organ specialties and acute care, it remains unclear if they are able to make the decisions concerning the management 


\section{Training the} future physician

Flexible portfolio training Less-than-full-time training Dual drivers towards generalism and sub specialism

\section{The future workforce \\ Multidisciplinary team care \\ Wider remit of allied health professionals \\ Flexible (including less-than- full-time) working patterns}
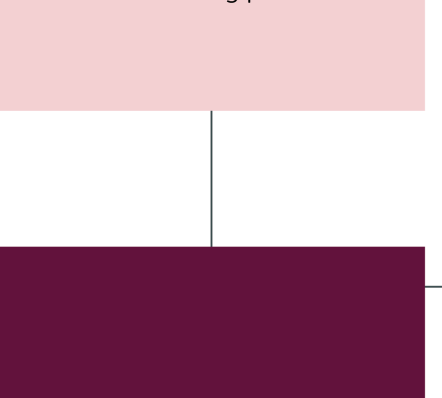

The future physician

\section{The future of health technology}

Virtual wards and clinics

Machine learning aid

in diagnostics

Enhanced patient monitoring

Machine-assisted procedures

\section{The future patient} Increase in complex, chronic conditions

Increased patient awareness and autonomy

Personalised healthcare approach

Fig 1. Key themes facing the physician today which will impact on their future role.

of complex multi-morbid patients. ${ }^{4}$ The current changes in physician training, which will increase the number of specialties dual training with general internal medicine (GIM) and place increased emphasis on these generalist skills, were designed to equip the medical workforce with the skills to care for the patients of tomorrow. ${ }^{5}$ The need for this has never been greater than now, with doctors having to train and retrain on an expedient basis in order to deliver patient-centred rather than system-based care.

Integrated teams of physicians, MAPs and allied health professionals will be required to deliver the complex, personalised care of the future. Novel ways for teams to work together, such as that espoused by the Buurtzorg model of care, will be required in order to break down the silo mentality within each profession. ${ }^{6}$

This innovative and cost-effective Dutch model provides for patients with multimorbid conditions in the community, with small groups of nurses given the autonomy to provide integrated clinical and social care. Such models, while needing some reform to work in the health and social care system here, will allow care to focus on the rapidly changing needs of our patients.

\section{The future patient: an equal partner in the management of complexity}

Over the last 30 years, despite rising demand, inpatient hospital beds have halved in number, facilitated by marked reductions in inpatient length of stay. ${ }^{7}$ Community and ambulatory care services have improved and increased, and these models of care will spread to other areas of practice. ${ }^{8}$ However, the ageing population, the greatest demographic driver of healthcare system change, and the increased multi-morbidity and complex care requirements this brings, will more than offset any efficiency savings that can be made in healthcare delivery. ${ }^{9}$

Not only must the workforce adapt to an older, multimorbid population, but so too must the evidence base for care. Historically, the National Institute for Health and Care Excellence (NICE) wrote guidance for single conditions in isolation. However, in response to the recognition that particular conditions commonly affect patients together (eg diabetes, hypertension, ischaemic heart disease, heart failure and chronic kidney disease), NICE is starting to develop guidance for condition combinations. ${ }^{10}$ A number of professional societies have followed suit, creating guidelines that recognise the ramifications of chronic conditions and their treatments on other organ systems. Such guidance will greatly facilitate the management of multi-morbidity, particularly by junior physicians or non-physicians. The internet, patient forums and societies have allowed patients to communicate with each other about care and have rightly increased expectations of their doctors' knowledge of current treatment and research. Patient experts have taken ownership of their conditions and in many instances help set the direction of care, both in the clinical and research setting, thanks to the encouragement of patient and public involvement. Patients will increasingly expect decision-making to be shared and personalised care to become the norm, with doctors as facilitators and advisers in their health management. In times of uncertainty, 
though, patients and the public will continue to expect doctors to take the lead on their management, and as the current situation has demonstrated, the public has a deep appetite for the view and expertise of the physicians in all aspects of their health.

\section{The future healthcare organisation: from cure to prevention via integration}

It is universally recognised that primary, secondary and adult social care will need to develop a more seamless working relationship to tackle the complex health needs of the population and deliver value-based healthcare. Integrated care partnerships will allow services to focus on the needs of the population, and ensure that care for particular groups of people are considered in the round, rather than from the perspective of a particular provider. ${ }^{11}$ It can also facilitate the integration of health improvement and prevention, and allow a true population approach to improving health outcomes. This will be facilitated by an increased availability of technology allowing joinedup care between healthcare systems, but current healthcare organisations will need to adapt if such a change of focus is to occur. ${ }^{12}$

In parallel to these changes, the inexorable trend towards sub-specialism will continue, driven by the accelerating pace of research in every specialty. With the call for more personalised care, the advances in complex therapeutics and the roll-out of genomics, it is not feasible to expect the generalist to keep upto-date in every area even with digital knowledge resources, and there will be a need for such expertise in all care providers, not just teaching hospitals and specialist centres. Formalised networks of providers and specialists will be required, with novel technologies and ways of working employed to connect patients in need of subspecialist input quickly and locally.

These dual trends towards subspecialism and generalism can seem contradictory, even in conflict. However, an older multimorbid population will, in an age of advancing therapeutic options, require both generalist physicians to take a personalised, holistic approach, and subject specialists with technical skills and specific knowledge. For instance, the care of patients with advanced cancer has recently been revolutionised by checkpoint inhibitor therapies, the use of which is expanding rapidly. However, side effects include severe immune-related adverse events, affecting multiple organ systems and presenting to different specialties as well as general medical services. Oncologists and other specialties are developing more collegial ways of working to provide the timely specialist input required to manage these complications and deliver good outcomes for patients. ${ }^{13}$

More immediately, the SARS-CoV-2 infection has been affecting adults of all ages and with older patients, their comorbidities will require specialists to play an important role in their care during this pandemic. This has been recognised by the specialty-specific organisations as they have moved quickly to provide systemspecific guidance both to the specialty and general physicians.

\section{The future of health technology: restoring the humanity to medicine?}

The use of artificial intelligence (AI) and robotics within the healthcare sector will exponentially increase, radically altering the working lives of future physicians. While clinical history and physical examination remain the cornerstones of clinical medicine, machine learning has already been incorporated into diagnostic pathways, and this trend will see AI platforms work alongside doctors in clinical decision-making. Recent work in ophthalmology, for example, has developed computer models that have expertlevel sensitivities for specialist referral for a number of sight threatening retinal diseases. ${ }^{14}$ Clinical decision trees exist for many conditions already, and access to information on various clinical questions in real time within the consultation is now possible. The role of AI programmes as 'clinical assistants', reminding doctors of factors to consider and providing ready access to information, is close, as both natural language processing and AI systems rapidly develop, and will allow the generalist to apply expert knowledge and guidelines from outside their specialty. ${ }^{15}$

Indeed, the Topol review suggests natural language processing use will be widespread by 2030 and automated image interpretation and predictive analytics by $2035 .{ }^{16}$

Technological developments will change procedural tasks, both through replacement of skilled practitioners in some fields with automation and robotics, and through changes to training. ${ }^{17}$ Complex techniques that historically took years to perfect can be repeated rapidly in safe, virtual-reality environments. ${ }^{18}$ While such methods are no replacement for real world experience currently, these platforms will become increasingly realistic, and may reduce training time. This will be all the more important as doctors will be required to perform the most challenging, high-risk procedures in complex or unusual cases, to lead research and development of new techniques, and to train and supervise other clinicians.

Diagnosis, management and procedural skills, historically the remit of the physician, are being democratised and delegated to technology or other professional groups. With this trend, the role of the consultant physician becomes more nuanced. As with procedural skills, doctors will focus on the unusual and the complex, the training and supervision of others, leadership, quality improvement, and research. In their patient-facing roles, consultant physicians will be cognitively freed from the protocols, checklists, and algorithms that consume so much mental bandwidth, enabling them to focus on the most complex aspect of healthcare: the patient's humanity at the centre of it all. In short, doctors will be experts in complexity: the procedural, diagnostic, therapeutic and human aspects of the job that cannot be delegated to others or replaced by machines. As difficult as it is to model accurately, we outline what the schedule of a general medical physician might entail in the future (see Box 1).

\section{Training the future physician}

The postgraduate training of doctors will need to reflect this diversity in remit. It will be a challenge to balance the desire and need of physicians to perform procedures, while ensuring the system has enough trained allied healthcare professionals to deliver the service effectively.

Doctors will expect more flexible training pathways, and lessthan-full-time training is becoming an increasingly popular choice for personal and professional reasons. ${ }^{19}$ This concept needs to be expanded within physician training alongside the flexible portfolio training posts piloted this year. ${ }^{20}$ To encourage uptake of these posts, the research, informatics, leadership, education and quality improvement (QI) skills developed will need to remain transferrable and count toward the Certificate of Completion of Training. This diversity of skills within the consultant workforce will 


\section{Box 1. A day in the life of a consultant gastroenterologist in 2040}

\subsection{0am Handover from the Hospital at Night team}

All patients requiring urgent gastroenterology review are automatically flagged by the electronic patient record (EPR) system. We discharge Philip, a man admitted yesterday for a gastrointestinal bleed. The EPR auto-generates the discharge medication and a discharge letter using natural language processing of the clinical records, which we review and edit in real time. He starts packing immediately - the discharge summary will be with him and his GP by email instantly, and the medication will be delivered to his home that afternoon.

\section{$8.30 \mathrm{am}$ Multidisciplinary team meeting to discuss inpatients}

\subsection{0am Inpatient ward round}

Ward round maps plot the most efficient route to the patients, factoring in physiological derangement and potential discharges. The integrated analytics system in the EPR picks up trends in blood tests, flagging concerning trends and suggesting additional tests and interventions based on Trust policies. The EPR is linked to guidelines and evidence, and pulls through key links in real time, augmenting decision-making and learning on the round.

\section{$12.00 \mathrm{pm}$ Virtual ward review and urgent virtual clinic}

Many patients are managed via the hospital at home set-up and we review them with the community physician associate and nurse via teleconference, with live physiological parameters from the patient from point-of-care sensors linked to the EPR via the internet. One patient requires specialist renal input - we arrange a video consultation with us, the patient and the specialist.

\section{$1.00 \mathrm{pm}$ Lunch}

\section{$1.30 \mathrm{pm}$ Administration}

I catch up with my paperwork - there isn't much to do. Referrals are processed by the EPR artificial intelligence system, which then suggests appropriate clinics and investigations for the patients for me to approve. Lab and endoscopy results are also processed by the EPR AI, with letters to patients and GPs created based on my previous correspondence, which I edit as required.

\subsection{0pm Endoscopy list}

This is a blended endoscopy session. First, I review the AI interpretation of the upper GI and colonoscopy capsules performed yesterday and approve reports for dispatch. There are two clinical and nurse endoscopist lists in parallel with my therapeutic procedure list. Real-time image analysis performs enhanced polyp detection and allows automatic interpretation of pit pattern and morphology to decide whether resection is necessary, assisted by endoscopic robotics for larger high-risk polyps. Endoscopy reports are generated automatically based on the findings.

\subsection{5pm Acute Medical Unit handover}

$5.00 \mathrm{pm}$ Review of patients from the acute medical floor Physician associates and advanced care practitioners work alongside medical trainees assessing patients. The hospital's electronic communication system allows for online specialty advice and input, facilitating patient flow through the unit.

\subsection{0pm Handover to the Hospital at Night team}

Having done 12 hours work, I hand over to the off-site consultant covering the night. It's recognised my decision-making after 12 hours isn't optimal and being woken at night impacts my performance the next day (when I'm only in for a morning ward round). be critical if the vision of the future of healthcare is to be achieved. These reforms to postgraduate training will only be possible with adequate medical staffing. Predicting these workforce needs is complex, particularly given the growing role of MAPs and technology. While staffing remains so compromised in many services, this vision for physician training will remain in tension with workforce needs.

Research in the future will continue to be led by clinical academics with patient and public involvement, and doctors are uniquely placed to direct research from bench to bedside according to their patients' needs. The pace of research, led primarily by clinical academics, in the face of the current pandemic has led to the development of rapid diagnostic testing and development of trials for a candidate vaccine within a couple of months. ${ }^{21}$ The collaborative links between academics and industry established as a result of urgent need will need to be fostered by future clinicians.

The excellent academic training programmes currently available should continue, with additional opportunities for all doctors to be exposed to research, and a clear pathway to train tomorrow's academic leaders. The RCP report Research for all addresses the barriers faced by non-academic clinicians in conducting research and sets out good practice to ensure that all clinicians have the opportunity to carry out research in some capacity. ${ }^{22}$ This will need to become more widespread, probably through the delivery of more flexible portfolio training posts focused on research.

\section{The future in light of the SARS-CoV-2 pandemic}

The SARS-CoV-2 pandemic may be a once-in-a-lifetime crisis, but it will leave a lasting impact on the role of doctors at all stages in their training, from final year medical students, who will find themselves on the wards in a few months without the reassurance of their finals and shadowing, through to recently retired doctors, taking up clinical practice again to bolster the ranks of their overstretched colleagues. The flexibility of the workforce going forward will be key and the ability of doctors to practice across the breadth of their knowledge will be crucial. The upskilling of genera physicians in critical care and the reskilling of specialty physicians in acute medicine has been progressing at pace across secondary care, while multispecialty teams, with a blend of physicians and surgeons, have been deployed in some clinical service units to provide a timely and varied service. Elsewhere, doctors with key organisational and leadership skills have implemented radical structural changes in short time to create the capacity sorely needed both in the primary and secondary healthcare setting. The links formed across specialties during this time need to be maintained to deliver the personalised healthcare espoused in the 
NHS Long Term Plan..$^{23}$ The implementation of social distancing and shielding of vulnerable groups in the population has required the use of telephone and video clinics, which will be refined and developed in the next several months. Doctors will need to harness this form of consultation, which provides new opportunities for delivering patient care in their own homes.

The SARS-CoV-2 pandemic will continue to test the capacity of the NHS for the next 1-2 years at least, and it is difficult to predict the state of the physician workforce when the crisis ends. With the attention of policy makers, the media and the public on doctors, we have a unique opportunity to demand and lead the changes we need to make in order to prepare the physician for the future.

\section{Conclusion}

Social, technological, and scientific progress is reforming the role of the physician at breakneck speed. The role of the physician will change fundamentally over the coming decades, driven by how our patients, knowledge, workforce, and technology change around us. The SARS-CoV-2 pandemic has thrust many of these changes upon the physician workforce faster than any could have predicted, with all in healthcare finding their role and place in these new ways of working. Currently we are training physicians with the skills needed in the present, a skillset which, we believe, will leave doctors underequipped to be the physicians of the future. We can do better - we must do better - if we are to deliver on the promise on personalised, high-quality, holistic patient care.

\section{References}

1 Health Education England. Future Doctor: What do the NHS, patients and the public require from 21st-century doctors? HEE, 2019. Available from: www.hee.nhs.uk/our-work/future-doctor.

2 General Medical Council. GMC-regulated credentials for doctors. GMC, 2019.

3 NHS Improvement. Interim NHS people plan. NHS, 2019.

4 Mcdonnell A, Goodwin E, Kennedy $F$ et al. An evaluation of the implementation of advanced nurse practitioner (ANP) roles in an acute hospital setting. J Adv Nurs 2015;71:789-99.

5 General Medical Council. Generic professional capabilities: guidance on implementation for colleges and faculties. GMC, 2017.

6 Laloux F. Reinventing organizations: A guide to creating organiza tions inspired by the next stage in human consciousness. Nelson Parker; 2014
7 The King's Fund. NHS hospital bed numbers: past, present, future. Kings Fund, 2017 (updated 2020). www.kingsfund.org.uk/publications/nhs-hospital-bed-numbers.

8 Royal College of Physicians. Acute care toolkit 10: Ambulatory emergency care. RCP, 2015.

9 Government Office for Science. Future of an Ageing Population. Government Office for Science, 2016.

10 National Institute for Health and Care Excellence. Multimorbidity: clinical assessment and management. Guideline NG56. NICE, 2016.

11 Ham C. Making sense of integrated care systems, integrated care partnerships and accountable care organisations in the NHS in England. The King's Fund, 2018. Available from: www.kingsfund. org.uk/publications/making-sense-integrated-care-systems.

12 Buck D, Baylis A, Dougall D and Robertson R. A vision for population health: towards a healthier future. King's Fund, 2018.

13 Haanen J, Carbonnel F, Robert C et al. Management of toxicities from immunotherapy: ESMO Clinical Practice Guidelines for diagnosis, treatment and follow-up. Ann Oncol 2017;28(suppl 4): iv119-iv142.

14 De Fauw J, Ledsam JR, Romera-Paredes B et al. Clinically applicable deep learning for diagnosis and referral in retinal disease. Nat Med 2018:24:1342-50.

15 Demner-Fushman D, Chapman WW, McDonald CJ. What can natural language processing do for clinical decision support? J Biomed Inform 2009:42:760-72.

16 Topol E. The Topol review: preparing the healthcare workforce to deliver the digital future. Health Education England, 2019.

17 Wright JD. Robotic-assisted surgery balancing evidence and implementation. JAMA 2017;318:1545-7.

18 Kerr R. The future of surgery. Bull R Coll Surg Engl 2019;101:264-7.

19 Health Education England. Interim Evaluation of Emergency Medicine Trainees LTFT Pilot 2017-18. HEE, 2018.

20 Royal College of Physicians. Flexible portfolio training. RCP, 2019 Available from: www.rcplondon.ac.uk/projects/ flexible-portfolio-training.

21 COVID-19 vaccine development - Oxford Vaccine Group [Internet]. Available from: www.ovg.ox.ac.uk/news/ covid-19-vaccine-development.

22 Royal College of Physicians. Research for all: Sharing good practice in research management. RCP, 2017.

23 NHS England. The NHS long term plan. NHS, 2019.

Address for correspondence: Dr M Roycroft, AO Admin Corridor, Robert Hadfield Wing, Northern General Hospital, Herries Road, Sheffield, S5 7AU.

Email: matthew.roycroft@nhs.net 